\title{
Development and standardization of tinnitus handicap inventory in Kannada
}

\author{
Thomas Zacharia \\ Priyanka Vas Naik ${ }^{1}$ \\ Shwetha Sada \\ Jensy Gangan Kuniyil ${ }^{1}$ \\ Vikas Mysore Dwarakanath²
}

\begin{abstract}
Introduction: Tinnitus Handicap Inventory (THI) was developed in English to quantify the handicap caused due to tinnitus. According to a survey conducted by USA, 40 million people are affected by tinnitus. Objectives: India being a multilingual country needs to develop and standardize THI in Indian languages. The present study concentrates on Developing and Standardizing THI in Kannada. Materials and Methods: Questions from English version of THI were translated and modified by two professionals who have a degree of MA Kannada. Translated inventory was given to 50 native Kannada speakers for the familiarity check \& the most familiar sentences were included in the study. Tinnitus questionnaire was then administered, followed by the Kannada version of THI on 140 patients and total scores were obtained. Results: Analysis revealed $14 \%$ of the patients fall under the category of Slight, $38 \%$ under Mild, $26 \%$ under Moderate, 16\% under Severe and 6\% under Catastrophic group. An item total correlation and Cronbach-alpha test was administered to examine the reliability measures and the scores were 0.883 and 0.885 on standardized item. Conclusion: Scores of Cronbach-alpha test shows that Kannada version of THI is a standard and reliable tool for measuring the handicap caused by tinnitus and can successfully classify individuals on a severity basis.
\end{abstract}

Keywords: quality of life, questionnaires, tinnitus.

\footnotetext{
${ }^{1}$ Department of Speech and Hearing - Father Muller Medical College - Mangalore - AC - India. E-mail: christin.zacharia@yahoo.com.

E-mail: priyankaedith@gmail.com. E-mail: shwetha192@gmail.com. E-mail: aslpjensy@gmail.com

${ }^{2}$ Department of Speech Language Pathology - All India Institute of Speech \& Hearing - Mysore - India. E-mail: vikasmdsIp@gmail.com

Institution: Father Muller Medical College.

Send correspondence to:

Thomas Zacharia.

Department of Speech \& Hearing, Father Muller College of Speech \& Hearing, A unit of Father Muller Charitable Institution. Kankanady, Mangalore. Karnataka, India. 575002. Phone: 07204546153

Paper submitted to the ITJ-SGP (Publishing Management System) on December 17, 2012;

and accepted on January 17, 2013. cod. 110.
} 


\section{INTRODUCTION}

Tinnitus is a common yet poorly understood disorder ${ }^{1}$. According to the data obtained from the National Center for Health Statistics, tinnitus is more commonly seen in males than females and increases in prevalence with advancing age. The diagnosis and rational management of tinnitus is one of the legacies of the past millennium, which the medical profession has carried unsolved to this century ${ }^{2}$. Tinnitus is the perception of sound in the absence of an external acoustical stimulus ${ }^{3}$. In many cases it is not a serious problem, but rather a nuisance that eventually resolves.

Recent interest has surfaced in symptom - specific handicap outcome measures for many audiological and otological diseases ${ }^{4}$. There are two important aspects of symptom - specific evaluation that is generally done by a valid and reliable inventory ${ }^{5}$. Initially, the self-assessment tool assesses the severity of symptoms and provides objective data about the severity of the symptomand later, the evaluation allows investigators to accurately assess and compare the pre-treatment and post-treatment periods.

A symptom specific assessment of many otological disorders will aid in understanding the severity of the symptom and effects of this on routine life. A Tinnitus Handicap Inventory (THI) was developed in order to measure the handicap caused by tinnitus. THI is a method of quantifying the impact that tinnitus has on an individual's daily living conditions. The THI was developed by the British Association of Otolaryngologist, Head and Neck surgeons, which consists of 50 questions ${ }^{6}$. Later, a modified beta version with 25 questions was developed by Newman et al..$^{5}$ which is in the form of a self-assessment report that a person suffering from the condition can fill in. The self report has been developed subsequent to previous studies that have thrown up reliable data that can be used to classify tinnitus into different grades according to the degree to which the tinnitus has affected a subject. THI consists of 25 questions under the 3 sub-scales of emotional, functional and catastrophic. The THI beta version is one of the best and most standardized tinnitus measuring tools and has got a good reliability, validity and a good internal consistency to administer on tinnitus patients.

The English version of THI which is shown in Appendix 1 was the only tool available for quantifying the handicap caused by tinnitus. India being a multilingual country with a multicultural background, there is no such inventory available in any of the Indian languages other than in Tamil. Being a developing country, the population who can read and understand English is less. Most of the population of India still depends on the regional language for communication. In such circumstance administering these inventories becomes difficult and sometimes impossible too. As this inventory quantified the alterations of the daily life of an individual due to tinnitus and due to the non capability of multilingual Speech \& Hearing professionals to translate accurately the English version of $\mathrm{THI}$ to the regional languages and to avoid communication interpretation, there is a need to develop and standardize the Tinnitus Handicap Inventory in Indian languages. Hence this study focused on developing THI in Kannada and to standardize the same.

Table 1. Rating scale of tinnitus handicap inventory.

\begin{tabular}{cc}
\hline THI SCORE & Degree of the condition \\
\hline $0-16$ & Slight \\
$18-36$ & Mild \\
$38-56$ & Moderate \\
$58-76$ & Severe \\
$78-100$ & Catastrophic \\
\hline
\end{tabular}

\section{SUBJECTS AND METHODS}

\section{Development of the Inventory}

The beta version of $\mathrm{THI}$ developed by Newman and Jacobson in 1996 consists of 25 questions under the 3 sub scales of emotional, functional and catastrophic. For the present study, the English version of THI was translated to Kannada using the standard Translation-back-translation method (Bardley, 1994) by two professors who have a degree of MA in Kannada. The translated form of the inventory was given to 50 native Kannada speakers who were exposed to Kannada language or had first language as Kannada, who were able to read and write Kannada, for the content validation. The speakers were given a five-point rating scale to grade the items from very familiar to non-familiar. Later, all the 50 filled inventories were assessed to find out if all the words and the meaning of the questions were understood by the Kannada speakers. The questions which were rated as 1 or 2 were selected for the inventory and any questions with ratings over 3 were re-framed according to the familiarity of the speakers. 


\section{Participants}

Individuals who were suffering from tinnitus with or without any otological diseases were selected for the study. Participants were interviewed briefly regarding tinnitus and those who complained tinnitus for more than 3 months were included for the further procedures, so that, they can quantify accurately the physical, emotional and catastrophic impacts of tinnitus on their daily acvtivities. Individuals with any significant neurological history, history of psychiatric or psychological counseling with regard to their tinnitus were excluded from the study. The Kannada version of THI was administered on a total of 140 patients of age range 24 to 75 with a mean age of 51.9 years with a standard deviation (SD) of 19.1. Out of 140 patients $86(61.5 \%)$ were males and 54 (38.5\%) were females. All the participants underwent assessment by an otorhinolaryngologist and complete audiological evaluation prior to the administration of the inventory.

\section{Procedure}

Tinnitus questionnaire was administered on the participants using a structured interview by the clinician to have a record of the demographic details and to cognize the type, nature, and the lateralization of the tinnitus, factors that affect tinnitus, presence of hearing loss, and the concern about the tinnitus.

Following the administration of the tinnitus questionnaire, the participants were instructed regarding the self - assessing Kannada version of THI, its importance and the system to grade it. Thereafter based on the subject's grading for all the questions, the total scores were calculated. For each questions in the Kannada version of THI, there are 3 options that the patient has to mark based on his/her perception of the problem namely YES, $\mathrm{NO}$, and SOMETIMES. A score of 4 was given for YES and a score of 0 was given for $\mathrm{NO}$ and a score of 2 was given for SOMETIMES. Once the subject finished rating the questions, they were grouped under 5 categories based on the severity that is Slight, Mild, Moderate, Severe and Catastrophic.

Among all the participants, $94 \%$ of the participants had a minimum qualification of SSLC and a maximum qualification of a degree and could fill up the Kannada version of THI without the help of the clinician. Rest percentages of participants were from a lower socio-economic status and could fill up the questionnaire only with the help of the clinician due to their inability to read and understand Kannada.

The obtained data were analyzed for statistical significance. Cronbach alpha test was administered to check for the internal consistency and reliability of the Kannada version of the THI and also an Item-Total Correlation was also carried out.

\section{RESULTS}

The results of the $\mathrm{THI}$ and the self assessing questionnaire were tabulated and are graphically represented below in Graph 1. The result shows that under emotional subscale, $28.8 \%$ of responses were for YES, $48.6 \%$ were for NO and $22.6 \%$ were for SOMETIMES. Under the functional subscale, $30 \%$ of responses were for YES and $50 \%$ of responses were for NO and $20 \%$ of responses were for SOMETIMES. Under catastrophic subscale, $28 \%$ of responses were for YES, $50.4 \%$ were NO and $21.6 \%$ of responses were for SOMETIMES.

After the complete analysis of the obtained data, the percentages of the patients coming under various severities were found out. $14 \%$ of the patients fall under the category of slight. $38 \%$ of patients fall under the category of mild, $26 \%$ of patients fall under the category of Moderate, and $16 \%$ fall under the category of severe and $6 \%$ fall under the category of catastrophic group which is represented in Graph 2.

The statistical measure (Cronbach alpha) score of 0.883 was obtained and on standardized item, a score of 0.885 was obtained. Thus, according to the Cronbach alpha score, the Kannada version of THI has got a good internal consistency and reliability to administer on the tinnitus patients in the Kannada population. Also a comparative study was carried out between the English version of THI and the Kannada version of THI. The overall Cronbach alpha score of both the THI versions and the individual scores for all the three subscales in both the versions were compared. An item-total correlation was also carried out for the Kannada version of THI.

\section{DISCUSSION}

The purpose of this study was to develop a handicap inventory in native language (Kannada), to obtain a standard measurement instrument for evaluating tinnitus in patients to apprehend the effects caused due to tinnitus. With the administration of Kannada THI it was found that, all the subjects could quantify the severity of tinnitus from slight degree to catastrophic degree, and thus fulfilled the aim of the study. The quality and intensity of tinnitus can affect daily routine of an individual which have an effect on the individual's psychological functioning ${ }^{7}$. Using the scores obtained by the Kannada version of THI, the 
Table 2. Kannada version of Tinnitus Handicap Inventory with associated rates and item - total correlation.

\begin{tabular}{|c|c|c|c|c|c|}
\hline SI. no & Items & Yes & No & Sometimes & $\begin{array}{l}\text { Item-total } \\
\text { correlation }\end{array}$ \\
\hline $1 \mathrm{~F}$ & Because of your tinnitus is it difficult for you to concentrate? & 48 & 74 & 18 & 0.63 \\
\hline $2 \mathrm{~F}$ & $\begin{array}{l}\text { Does the loudness of your tinnitus make it difficult for you to hear } \\
\text { people? }\end{array}$ & 35 & 86 & 19 & 0.62 \\
\hline $3 E$ & Does your tinnitus make you angry? & 27 & 85 & 28 & 0.66 \\
\hline $4 \mathrm{~F}$ & Does your tinnitus make you feel confused? & 41 & 67 & 32 & 0.56 \\
\hline $6 \mathrm{E}$ & Do you complain a great deal about your tinnitus? & 41 & 73 & 26 & 0.52 \\
\hline $7 \mathrm{~F}$ & Because of your tinnitus do you have trouble falling to sleep at night? & 31 & 72 & 37 & 0.44 \\
\hline $8 \mathrm{C}$ & Do you feel as though you cannot escape your tinnitus? & 21 & 80 & 39 & 0.47 \\
\hline $9 \mathrm{~F}$ & Does your tinnitus interfere with your ability to enjoy social activities? & 25 & 26 & 89 & 0.38 \\
\hline $10 \mathrm{E}$ & Because of your tinnitus do you feel frustrated? & 15 & 28 & 97 & 0.54 \\
\hline $13 \mathrm{~F}$ & Does your tinnitus interfere with your job or household responsibilities? & 27 & 87 & 26 & 0.47 \\
\hline $14 \mathrm{~F}$ & Because of your tinnitus do you find that you are often irritable? & 58 & 51 & 31 & 0.39 \\
\hline $15 \mathrm{~F}$ & Because of your tinnitus is it difficult for you to read? & 24 & 94 & 23 & 0.37 \\
\hline $16 \mathrm{E}$ & Does your tinnitus make you upset? & 32 & 78 & 30 & 0.58 \\
\hline $17 \mathrm{E}$ & $\begin{array}{l}\text { Do you feel that your tinnitus problem has placed stress on your } \\
\text { relationship with members of your family and friends? }\end{array}$ & 20 & 96 & 24 & 0.53 \\
\hline $18 \mathrm{~F}$ & $\begin{array}{l}\text { Do you find it difficult to focus your attention away from your tinnitus } \\
\text { and on other things? }\end{array}$ & 22 & 88 & 30 & 0.63 \\
\hline $19 \mathrm{C}$ & Do you feel that you have no control over your tinnitus? & 32 & 83 & 25 & 0.02 \\
\hline $20 \mathrm{~F}$ & Because of your tinnitus do you often feel tired? & 22 & 91 & 27 & 0.67 \\
\hline
\end{tabular}

The scores represented above are of the Kannada version of THI and the items are written in English language for easy understanding.

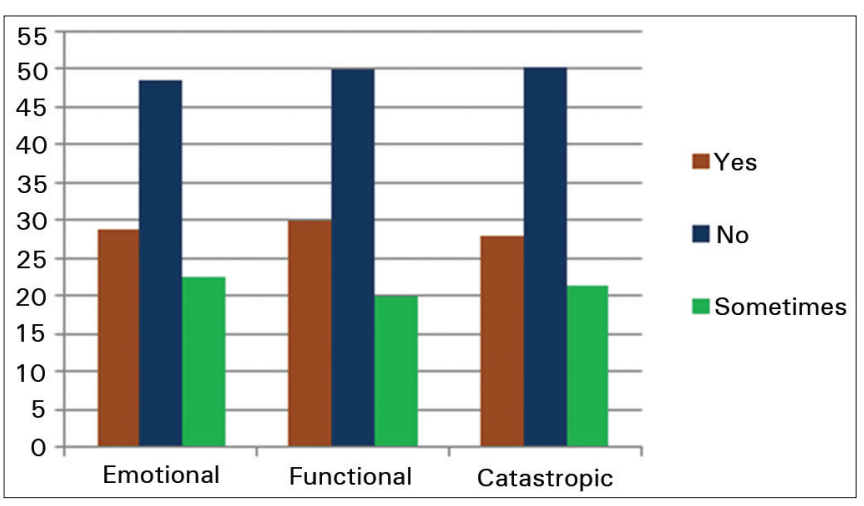

Graph 1. Percentage of participant's responses for emotional, functional \& catastrophic sub scales.

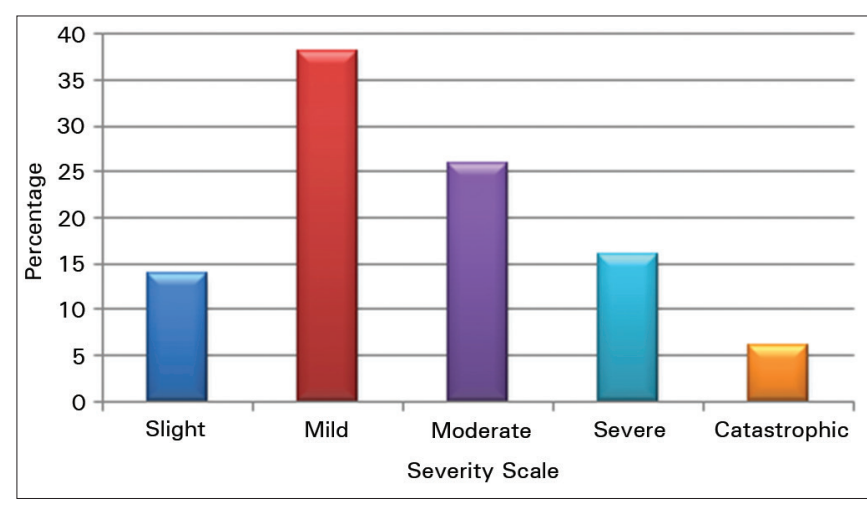

Graph 2. Percentage of subjects falling under different severities. 
subjects can be explained regarding the nature of the problem and severity of the problem, so that he/she will get a better understanding about the illness. If not his/her negative beliefs that are caused by opinions of friends and doctors that he has to live with it or it is incurable leads to emotional upsets and autonomic arousal phenomenon ${ }^{2}$.

From the study, we could observe that the perception of tinnitus handicap differs drastically between subjects as demonstrated by high values of standard deviations, which may be due to effective coping starategies ${ }^{5}$. Tinnitus assessment not only involves to match the tinnitus, ascertain the intensity of the tinnitus and to find out the intensity of wide band noise that can mask the tinnitus, but also involve administration of various inventories like $\mathrm{THI}$ to rate and assess the severity of tinnitus in order to help the clinician to counsel the patient as well as to know the level at which the management options should begin ${ }^{8}$.

Related to the emotional aspects, the Kannada THI allows assessing the relation between tinnitus and manifestation of stress, depression, anxiety \& frustration. With respect to the functional aspect, the Kannada THI allows to identify the relationship between tinnitus and activities involving concentration, hearing acuity, attention \& reading. The catastrophic aspect of Kannada THI allows identification of negative sensations produced by the tinnitus in the patient's lives such as intolerance, loss of control of the sensation.

The result of the current study shows good reliability and internal consistency as indicated by the Cronbach alpha test. The English version of THI has got a Cronbach alpha score of 0.93 with an Item-Total Correlation ranging between $0.22-0.77^{9}$. The Kannada version of THI has got an alpha score of 0.885 , with an Item - Total Correlation ranging between 0.02 - 0.67. While comparing the Cronbach alpha scores and Item-Total Correlation across the three subscales; emotional, functional and catastrophic, the English version of $\mathrm{THI}$ has got alpha scores such as 0.87 , 0.86 , and 0.68 and an Item-total correlation of 0.56 $0.82,0.27-0.76$, and $0.42-0.48$ respectively across emotional, functional and catastrophic subscales. In the case of Kannada version of THI, the alpha scores across emotional, functional and catastrophic are 0.78 , 0.79 and 0.68 and Item-total correlation of $0.44-0.66$, 0.37-0.67 and 0.02-0.48 respectively.

While comparing the Cronbach alpha scores of Kannada THI with the Turkish THI, the alpha score is 0.885 for Kannada THI and is 0.886 for the Turkish THI. When comparing the alpha scores across emotional, functional and catastrophic subscales among Kannada
THI \& Turkish THI, the scores are $0.78,0.79$ and 0.68 for Kannada THI and 0.87, 0.78 and 0.80 for Turkish version of THI respectively. A comparison was also carried out between the Italian versions of $\mathrm{TH}$ and found out that, Italian version has got a total alpha score of 0.91 and is 0.885 for the Kannada THI. Across the three subscales, the Italian $\mathrm{THI}$ has got alpha scores of 0.86 , 0.85 and 0.63 respectively across emotional, functional and catastrophic scales and is $0.78,0.79$ and 0.68 respectively for Kannada THI. When comparing with the Danish version of THI, it was observed that, it has got a total alpha score of 0.93 and across emotional, functional and catastrophic scales; the scores are $0.88,0.87$ and 0.74 respectively and on the other hand, the Kannada version of THI has got a total alpha score of 0.885 and across emotional, functional and catastrophic scales the scores are $0.78,0.79$ and 0.68 respectively.

Hence this inventory can serve as an effective tool to successfully classify the individuals into different grades according to the symptoms. This inventory could easily be understood to all the laymen and $94 \%$ of patients could fill the inventory without any help from the clinician. The English version of $\mathrm{THI}$ is currently used by most of the audiologists and otorhinolaryngologist to find out the severity of the problem in different patient population and found to be difficult to administer on native speakers of Karnataka.

Since the main focus of this study was to develop and standardize the THI into Kannada language, and to be administered on all Kannada patients who complain of tinnitus. Following all the analysis and standardization, the Kannada version of THI came out successfully for the evaluation of tinnitus and found to be reliable.

\section{CONCLUSION}

The main aim of this study was to develop and standardize the Tinnitus Handicap Inventory in Kannada; hence it can be administered on Kannada patients without difficulty. The Kannada version will help the patients with tinnitus to comprehend the severity and intensity of their problem and also considered as a reliable instrument for the verification of the impairment in the quality of life due to tinnitus. The recruited subjects for this research presented changes in their Quality of Life (QL) as regards to the functional, emotional and catastrophic aspects due to tinnitus. From the current study, it is clear that the Kannada version of $\mathrm{THI}$ is readily available, easy to understand and is a reliable tool for measuring the handicap occurring due to tinnitus. 
Appendix 1. The English version of Tinnitus Handicap Inventory.

\begin{tabular}{|c|c|c|c|c|}
\hline No & Items & Yes & No & Sometimes \\
\hline $1 \mathrm{~F}$ & Because of your tinnitus is it difficult for you to concentrate? & 4 & 0 & 2 \\
\hline $2 \mathrm{~F}$ & Does the loudness of your tinnitus make it difficult for you to hear people? & 4 & 0 & 2 \\
\hline $3 \mathrm{E}$ & Does your tinnitus make you angry? & 4 & 0 & 2 \\
\hline $4 \mathrm{~F}$ & Does your tinnitus make you feel confused? & 4 & 0 & 2 \\
\hline $5 \mathrm{C}$ & Because of your tinnitus do you feel desperate? & 4 & 0 & 2 \\
\hline $6 \mathrm{E}$ & Do you complain a great deal about your tinnitus? & 4 & 0 & 2 \\
\hline $7 \mathrm{~F}$ & Because of your tinnitus do you have trouble falling to sleep at night? & 4 & 0 & 2 \\
\hline $8 \mathrm{C}$ & Do you feel as though you cannot escape your tinnitus? & 4 & 0 & 2 \\
\hline $9 \mathrm{~F}$ & Does your tinnitus interfere with your ability to enjoy social activities? & 4 & 0 & 2 \\
\hline $10 \mathrm{E}$ & Because of your tinnitus do you feel frustrated? & 4 & 0 & 2 \\
\hline $11 \mathrm{C}$ & Because of your tinnitus do you feel that you have a terrible disease? & 4 & 0 & 2 \\
\hline $12 \mathrm{~F}$ & Does your tinnitus make it difficult for you to enjoy life? & 4 & 0 & 2 \\
\hline $13 \mathrm{~F}$ & Does your tinnitus interfere with your job or household responsibilities? & 4 & 0 & 2 \\
\hline $14 \mathrm{~F}$ & Because of your tinnitus do you find that you are often irritable? & 4 & 0 & 2 \\
\hline $15 \mathrm{~F}$ & Because of your tinnitus is it difficult for you to read? & 4 & 0 & 2 \\
\hline $16 \mathrm{E}$ & Does your tinnitus make you upset? & 4 & 0 & 2 \\
\hline 17E & $\begin{array}{l}\text { Do you feel that your tinnitus problem has placed stress on your relationship with members } \\
\text { of your family and friends? }\end{array}$ & 4 & 0 & 2 \\
\hline $18 \mathrm{~F}$ & Do you find it difficult to focus your attention away from your tinnitus and on other things? & 4 & 0 & 2 \\
\hline $19 \mathrm{C}$ & Do you feel that you have no control over your tinnitus? & 4 & 0 & 2 \\
\hline $20 \mathrm{~F}$ & Because of your tinnitus do you often feel tired? & 4 & 0 & 2 \\
\hline $21 \mathrm{E}$ & Because of your tinnitus do you feel depressed? & 4 & 0 & 2 \\
\hline $22 \mathrm{E}$ & Does your tinnitus make you anxious? & 4 & 0 & 2 \\
\hline $23 \mathrm{C}$ & Do you feel that you can no longer cope with your tinnitus? & 4 & 0 & 2 \\
\hline $24 \mathrm{~F}$ & Does your tinnitus get worse when you are under stress? & 4 & 0 & 2 \\
\hline $25 \mathrm{E}$ & Does your tinnitus make you feel insecure? & 4 & 0 & 2 \\
\hline
\end{tabular}

F represents items in the functional sub scale, E represents items in the emotional sub scale and C represents items in the catastrophic sub scale.

Appendix 2. The Tinnitus Questionnaire.

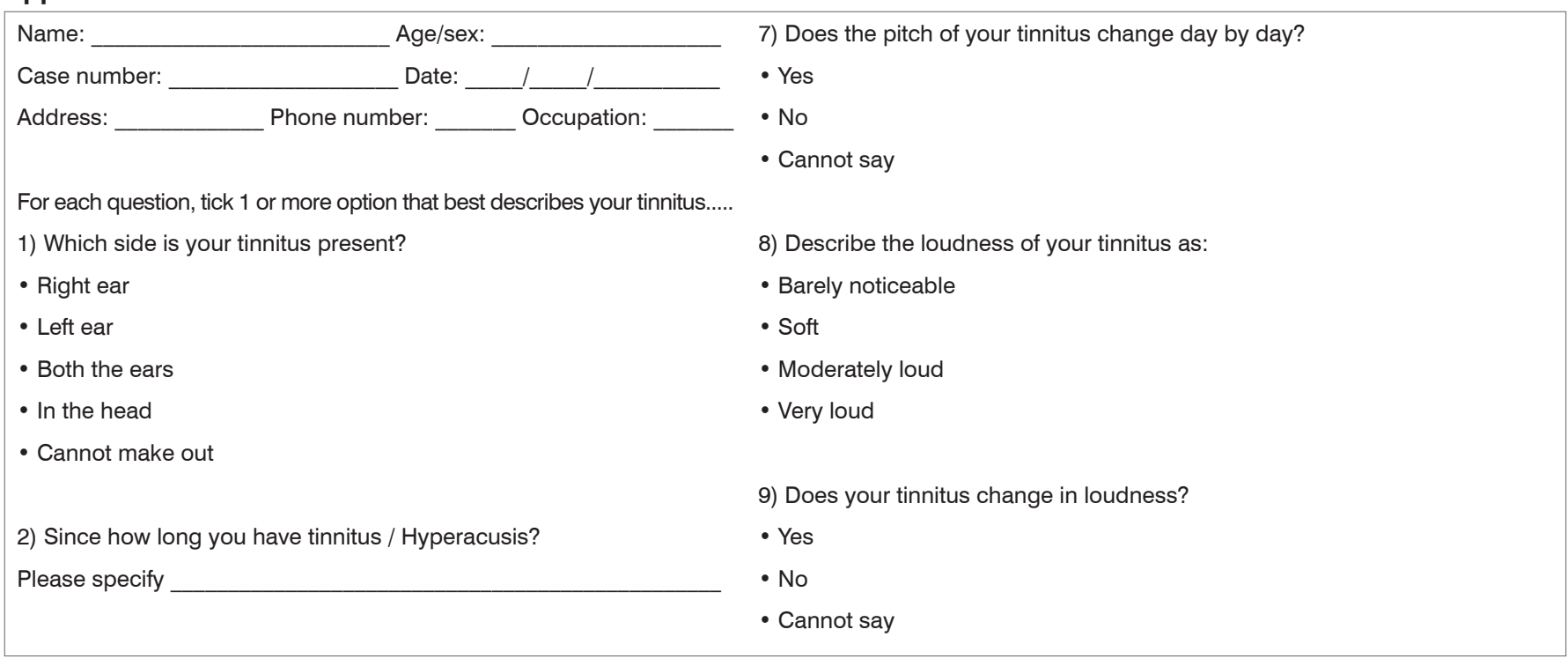




\section{Continued Appendix 2.}

\begin{tabular}{|c|c|}
\hline \multicolumn{2}{|l|}{ 3) Is your tinnitus: } \\
\hline - Intermittent & 10) Which of the following reduces or increases your tinnitus/ hyperacusis? Specify \\
\hline \multirow[t]{2}{*}{ - Continuous } & - Sleep \\
\hline & - Alcohol \\
\hline 4) When is your tinnitus / Hyperacusis worse? & - Noisy place \\
\hline - Morning & - Quiet place \\
\hline - Afternoon & - Tea/ coffee \\
\hline - Evening & - Wearing a hearing aid \\
\hline - Night & - Drugs/medicines \\
\hline \multirow[t]{2}{*}{ - Constant all the time } & - Any physical activity \\
\hline & - Smoking \\
\hline 5) How does your tinnitus sound? (Quality) & - Emotional or mental stress \\
\hline - Ringing & - Others please specify \\
\hline - Hissing & - Nothing makes it worse \\
\hline \multicolumn{2}{|l|}{ - Buzzing } \\
\hline - Ocean roar & 11) Do you have hearing loss? Which ear? \\
\hline - Humming & - Right ear \\
\hline - Pulsatile & - Left ear \\
\hline - Clicking & - Both the ears \\
\hline - Any other & - Cannot make out \\
\hline 6) Is the pitch of your tinnitus? & 12) Do you feel depressed? \\
\hline - Low & If so explain why \\
\hline \multicolumn{2}{|l|}{ - High } \\
\hline \multirow[t]{4}{*}{ - Cannot say } & 13) Rank (indicate by number) how much these concern you \\
\hline & $(1=$ least and $3=$ most $)$ \\
\hline & 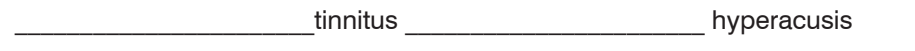 \\
\hline & hearing loss. \\
\hline
\end{tabular}

\section{REFERENCES}

1. Lockwood AH, Salvi RJ, Burkard RF. Tinnitus. N Engl J Med. 2002;347(12):904-10. PMID: 12239260 DOI: http://dx.doi. org/10.1056/NEJMra013395

2. Biswas A. An Introduction to Neurotology. Mumbai: Bhalani House Publishing; 2006. p.293-98.

3. Lockwood AH, Salvi RJ, Coad ML, Towsley ML, Wack DS, Murphy BW. The functional neuroanatomy of tinnitus: evidence for limbic system links and neural plasticity. Neurology. 1998;50(1):114-20. PMID: 9443467 DOI: http://dx.doi.org/10.1212/WNL.50.1.114

4. Aksoy S, Firat Y, Alpar R. The Tinnitus Handicap Inventory: a study of validity and reliability. Int Tinnitus J. 2007;13(2):94-8.

5. Newman CW, Jacobson GP, Spitzer JB. Development of the Tinnitus Handicap Inventory. Arch Otolaryngol Head Neck Surg. 1996;122(2):143-8. PMID: 8630207 DOI: http://dx.doi.org/10.1001/ archotol.1996.01890140029007
6. Kuk FK, Tyler RS, Russell D, Jordan H. The psychometric properties of a tinnitus handicap questionnaire. Ear Hear. 1990;11(6):434-45. DOI: http://dx.doi.org/10.1097/00003446-199012000-00005

7. Monzani D, Genovese E, Marrara A, Gherpelli C, Pingani L, Forghieri $M$, et al. Validity of the Italian adaptation of the Tinnitus Handicap Inventory; focus on quality of life and psychological distress in tinnitus-sufferers. Acta Otorhinolaryngol Ital. 2008;28(3):126-34.

8. Schmidt LP, Teixeira VN, Dall'Igna C, Dallagnol D, Smith MM. Brazilian Portuguese Language version of the "Tinnitus Handicap Inventory": validity and reproducibility. Braz J Otorhinolaryngol. 2006;72(6):808-10. PMID: 17308834

9. Baguley DM, Andersson G. Factor analysis of the Tinnitus Handicap Inventory. Am J Audiol. 2003;12(1):31-4. DOI: http://dx.doi. org/10.1044/1059-0889(2003/007) 